\title{
Evaluation of Property Scatter of Nickel Base Alloy IN 738 LC
}

\author{
Stefan Linn, Alfred Scholz and Christina Berger \\ Institut fuer Werkstoffkunde, Technische Universitaet Darmstadt, \\ Grafenstrasse 2, 64283 Darmstadt, Germany \\ corresponding author: linn@mpa-ifw.tu-darmstadt.de
}

\section{Key words: Nickel Base Alloy, Creep Behaviour, Scatter Band Assessment, Neural Networks}

\begin{abstract}
The nickel base alloy IN 738 LC is in use for gas turbine blades since more than 25 years. In high temperature creep testing the conventionally cast alloy exhibits a comparably large property scatter which requires high safety margins in design and dimensioning and subsequently causes an incomplete exploitation of the materials potential. The reasons for this property scatter were investigated and traced back to different influencing factors. Parallel to investigations on the microstructure of post-exposure material and conventional scatter band analysis, artificial neural networks were successfully applied to discover relations between the chemical composition of the individual melt and the position of the corresponding test results within the global scatter band. Recommendations for a lifting of the lower scatter band boundary and the mean curve are derived.
\end{abstract}

\section{Introduction}

Turbine blades with high thermal and mechanical loads are chiefly manufactured from cast nickel base alloys. These alloys exhibit in comparison to other metallic materials an outstanding high temperature strength. The processing of components is mostly made by investment casting which allows economic manufacturing with less machining of the final contour. Additionally the manufacturing of hollow blades is possible which, in conjunction with a thermal barrier coating and internal cooling, enables a considerable raise of the gas temperatures. Different solidification types are applied: Conventional casting (CC), which produces a polycrystalline microstructure with stochastic orientation, directionally solidification (DS) with grains in only one growth direction, and single crystal solidification (SX), where the entire component consists of only one single grain. DS and SX casting produces work pieces with increased mechanical strength requires considerably additional efforts and expenses. With respect to economy, conventional casting therefore is applied wherever possible.

An unfavourable property of conventional cast nickel base alloys is the scatter of the results of creep rupture tests and other relevant property data. This scatter is much larger than that which is observed with DS or SX and also wrought material. This paper deals with investigations on the reasons for the scatter and possible measures for its reduction. Within the project it was examined with respect to the scatter, how far the manufacturing process and the chemical composition of the master heat influence the creep rupture strength. 


\section{Possible Sources of Scatter}

The scatter band of creep rupture tests originates from different reasons. The first source of the scatter are minor differences between the master heats, which were taken for the fabrication of the specimens. The casting process itself, i.e. the temperatures of the melt and the mold, as well as the cooling down rate and other parameters may cause differences in the microstructure from specimen to specimen. Especially the grain size, the dendrite arm spacing and the preferred growth direction may differ slightly even within one cast cluster. The parameters of the following heat treatment may cause further deviations from the average condition of the microstructure. Finally the manufacturing of the specimen has a certain influence. The orientation of the blank within the component, deviations from the ideal shape, dents and scratches in the surface are additional sources for scatter. Small deviations from the nominal temperature and stress lead to longer or shorter test durations. Summarized, the test result is affected by a superposition of several influences. The properties of the master heat are passed down to the specimen, while the manufacturing process adds further individualities. The investigations described in this paper are concentrated on such parameters, which can either be controlled with justifiable efforts or which can be determined easily. Therefore only a small selection of parameters were dealt with. These are the chemical composition of the master heat, the HIP treatment and micro-porosity. Additional considerations were performed for the test temperature (resp. application temperature). The contribution of test uncertainties on the entire property scatter can be rated low, as far as the tests are performed in accordance with the applicable standards [1].

\section{Data Analysis}

The scatter band for a material grade is generated by assembling the mean curves of the single melts. Within the individual melts a certain degree of scatter will be observed but, however, the question arises, if there exists the possibility for positioning mean curves within the scatter band. It is self-evident, that manufacturers and users of the material are interested in those melts, whose properties are better than average. In order to find an answer to this demanding questions an analysis of correlations between input parameters and the creep rupture strength is necessary.

\subsection{Chemical composition}

With some restrictions, the chemical composition of melts belongs typically to those parameters, which are often properly documented. Modern analysing methods allow to identify the weight proportion of the alloying elements with sufficient accuracy. Common restrictions are a concentration on the main alloying elements and insufficient accuracy with elements of smaller percentages. The following explanations demonstrate that it is possible to calculate trends even with a limitation on 7 alloying elements.

Conventional scatter band analysing methods are well introduced since several decades. Timetemperature-parameter based models like the Larson-Miller-parameter allow, in combination with a stress function, to express the rupture time or time to a specific strain in creep testing as a function of test temperature and stress. A major advantage of such models is the possibility of a temperature or stress extrapolation, because the equations are based on Arrhenius- and Monkman-GrantApproaches [2]. Furthermore, a parameter identification is mostly possible by numerical regression. Artificial neural networks are an appropriate and contemporary attempt for the generation of a mathematical model for an "unclear" relation between input and output data. An overview on the application of Neural Networks in material science is given in [3], an example for a so-called "data 
mining" application in [4]. The basic idea of (artificial) neural networks is quite different from regression analysis, which is characterised by the attempt to find suitable constants that make a chosen equation represent a relation between input and output data. The neural network instead is trained to transfer a given input vector to a given output vector, whereas in general the transfer function itself is not predefined by the user. The system itself shall learn, which global transfer function is appropriate to map the input on the output

$t=f(\sigma, T, C r, A l, T i, M o \ldots)$

where the element descriptors $\mathrm{Cr}$ and $\mathrm{Al}$ etc. in (Eq. 1) represent the weight proportion in the alloy. A model of such type can deliver reasonable results only for the specific alloy whose data where used for training and only in the specified range of the specific alloy. The model shall furthermore not replace conventional approaches for scatter band evaluation. The absolute accuracy of the model is therefore to a lesser extend of interest than the relative evaluation of the specific alloys potential. Basically the model shall identify those "recipes" of the chemical composition, which are favourable for the creep rupture strength.

The preferred model takes into account the physical test parameters temperature and stress as well as the weight proportion of the elements $\mathrm{Al}, \mathrm{Cr}, \mathrm{Ti}, \mathrm{Ta}, \mathrm{Co}, \mathrm{Mo}$ and $\mathrm{W}$. The optimisation target is the logarithm of test time (rupture time). The net consists of only two layers, thereof one hidden layer. A number of 2 - 5 neurons in the hidden layer turned out to be suitable. The training data comprised about 400 single tests on specimens from 25 different melts (only CC, no HIP).

Fig. 1 displays isothermal curves for three specific melts together with belonging data points. The global trend is reproduced properly by the model, which is able to sort the melts in the correct order along with information on their chemical composition.

The verification of a neural net model is favourably executed by testing the ability to reproduce the relation between input and output data for a data set, which is completely different from the training data set. This is demonstrated in Fig. 2, where the capability of the model is tested versus a data set of melts with HIP treatment. At $650^{\circ} \mathrm{C}$ the isothermal curves are located too low in comparison to the data points, which is related to the positive influence of the HIPing but the global order of the curves is reproduced correctly.

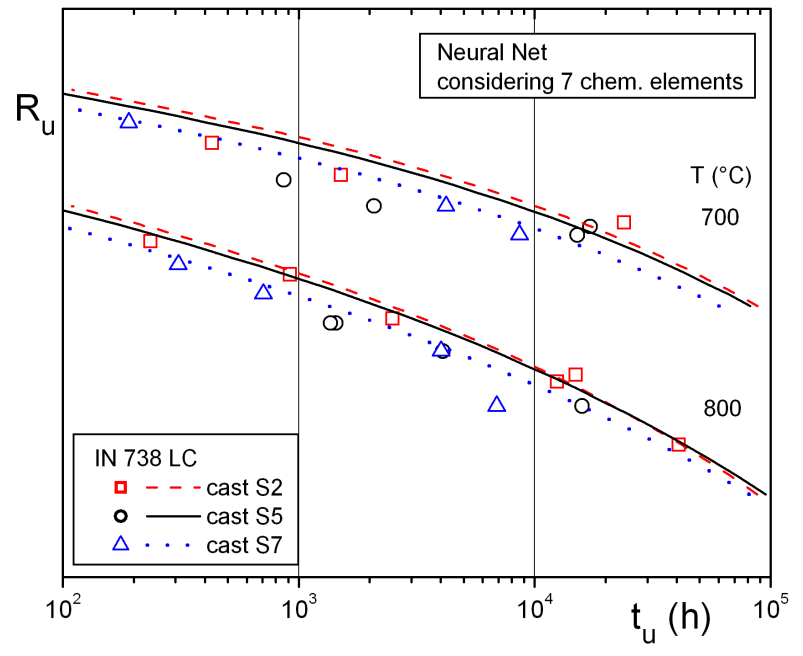

Fig. 1. Creep-Rupture Curves for three melts.

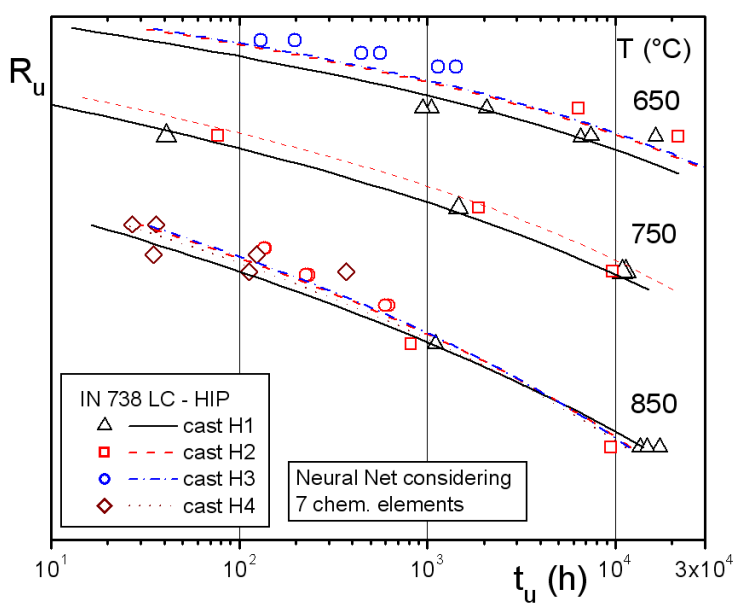

Fig. 2. Verification with HIP data set 
The contribution of the fluctuation of the chemical composition to the entire scatter of the creep rupture properties can be assessed as follows: The scatter band width with conventional evaluation $\mathrm{S}_{\text {conv }}$ is about 1.5 times larger than with neural network evaluation $\mathrm{S}_{\mathrm{NN}}$ (Eq. 2).

$$
S_{\text {conv }} \approx 1.5 \cdot S_{N N}
$$

Given that the total scatter is divided in a portion related to the chemical composition and a portion with miscellaneous reasons $\mathrm{S}_{\text {misc }}$, and that these portions can be added stochastically, eq. (3) provides the following estimation:

$$
S_{m i s c} \approx \sqrt{5 / 2} \cdot S_{N N}=1.1 \cdot S_{N N}
$$

Summarized, the fluctuation of the chemical composition between different melts is suitable to correlate partly with the total scatter but differences between the individual specimens are still dominant. It must be emphasised that the described model is clearly phenomenological and does not explicitly consider effects of the alloying elements on the microstructure. If, for example, certain compositions are prone to form undesirable phases like $\sigma$-phase, this tendency is implicitly included because specimens from such melts will fail in average earlier than other specimen.

\subsection{Hot isostatic pressing}

The effect of the HIP process in terms of a reduction of scatter and a shift of the lower boundary to higher values was proven without any doubt. The evidence was made with scatter band evaluation (Fig. 8) and considerations of the failure probability (Weibull - distribution, Fig. 9). Examined micrographs of specimens with HIP treatment did not show any casting porosity.

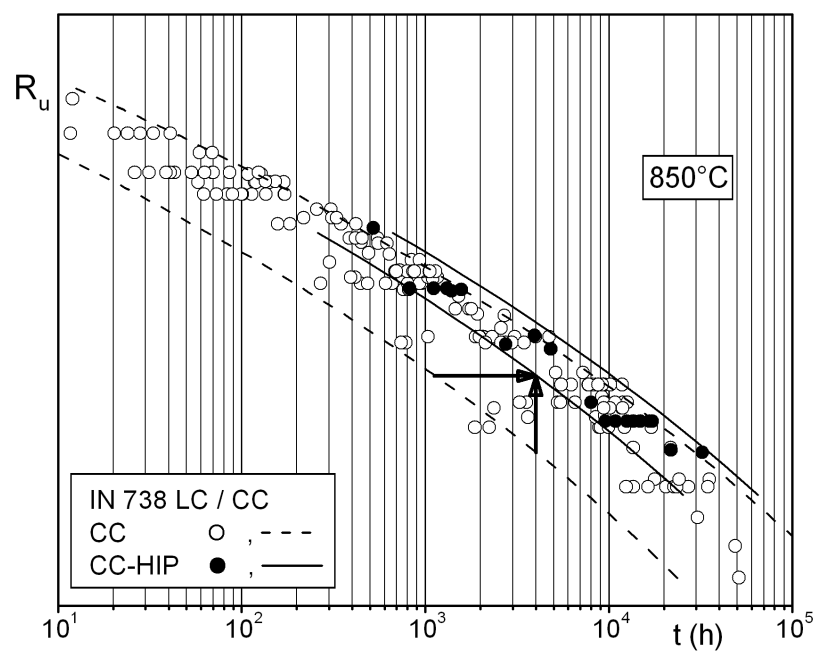

Fig. 8: Creep test results at $850^{\circ} \mathrm{C}$. Arrows indicate the shift of the lower scatter bound to longer times and higher stresses.

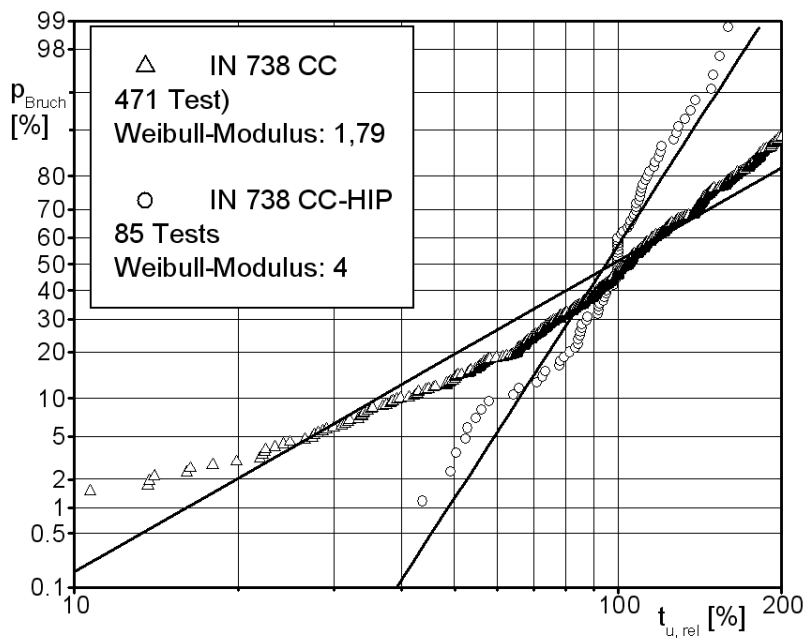

Fig. 9: Weibull-plot of Material with or without HIP-treatment. The larger weibull-modulus of HIP-Material indicates lower property scatter.

\subsection{Temperature dependence}

The observed scatter of rupture time in creep tests is dependent on the test temperature. In Fig. 10 a lower scatter with increasing temperature is clearly visible. Fig. 11 proves that this effect is related to the temperature and not to the applied stress: Within the transition region between different temperatures at the same stress level the scatter is smaller for the particular higher temperature. 


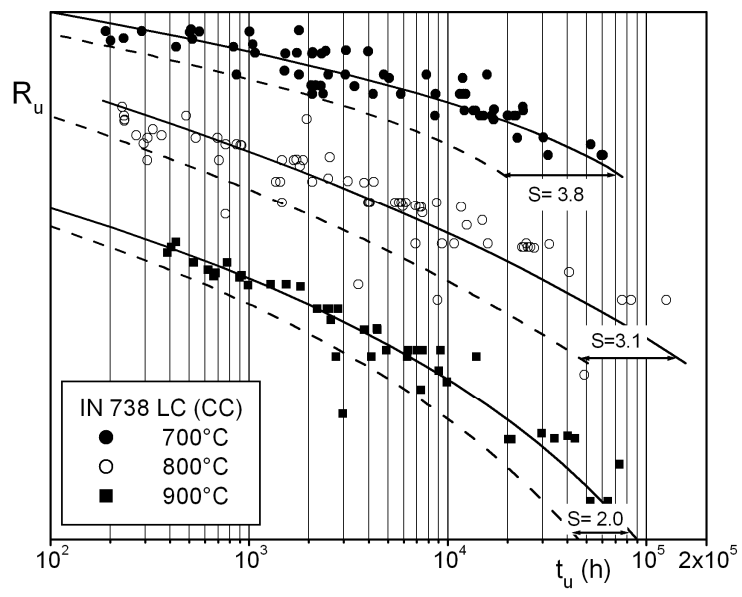

Fig. 10. Creep rupture curves.

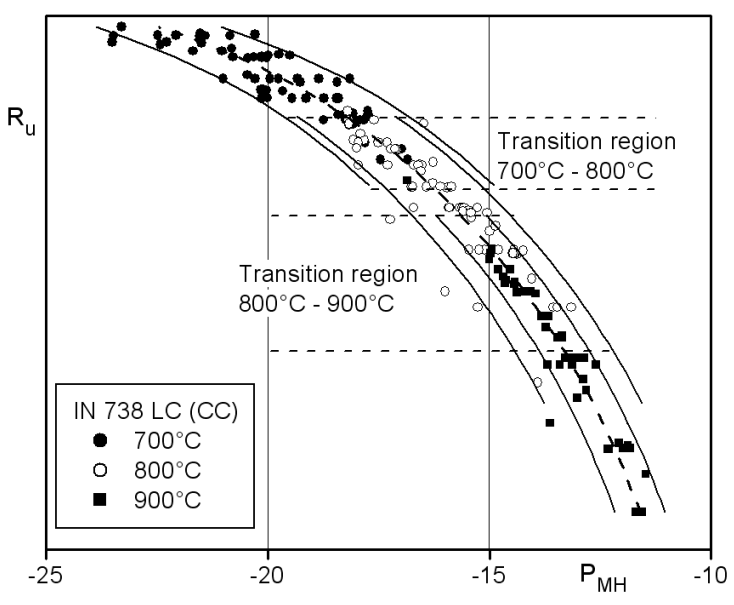

Fig. 11. Master-curve for the data in Fig. 10, based on Manson-Haferd-Parameter $\mathrm{P}_{\mathrm{MH}}$.

\section{Porosity}

The porosity of conventional cast alloy IN 738 LC turned out to be of essential importance for the creep rupture behaviour. Porosity becomes obvious both in micro graphs (ig. 12) and in the surfaces of fractured specimens.

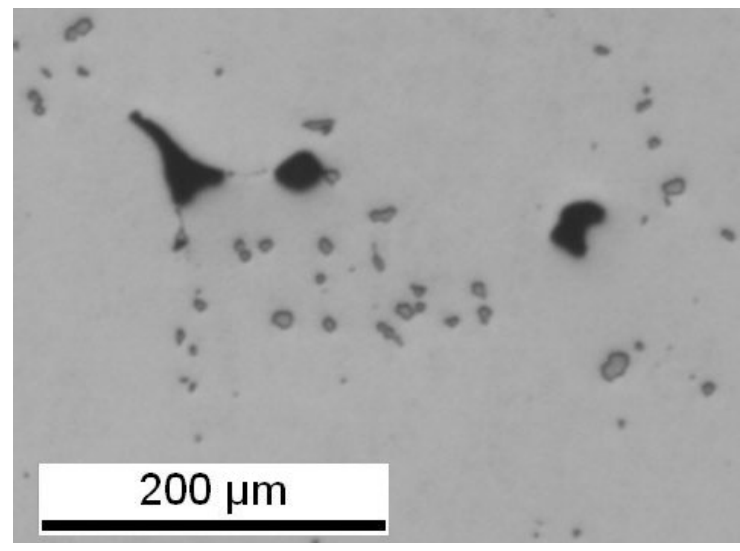

Fig. 12. Porosity in micro graph.

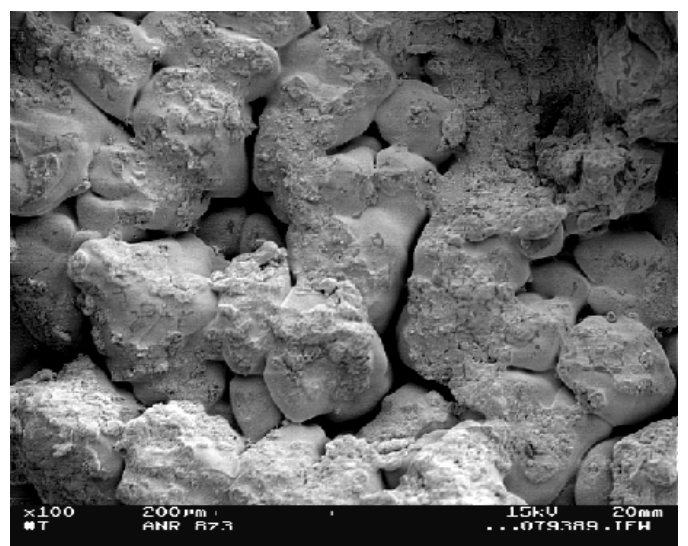

Fig. 13. Porosity in fractured surface (SEM- image)

The porosity as found in metallographic examinations of a variety of specimens was in the range from about $0.5 \%$ up to more than $1 \%$. These values are also reported in literature [5] for IN 738 LC. SEM-images of the ruptured surface of all specimens proved that to a certain extend porosity was involved in the crack process. A plot of the standardised creep rupture time versus the porosity indicates a clear trend (Fig. 14). A high porosity is statistically responsible for a shorter lifetime in creep testing. The positive effect of a HIP treatment can widely be explained by removing the porosity. Beyond that, porosity is suspected to have an significant impact on crack initiation. The crack propagation in course grain alloys exhibits sometimes a discontinuous progress and may cause additional scatter [6]. 


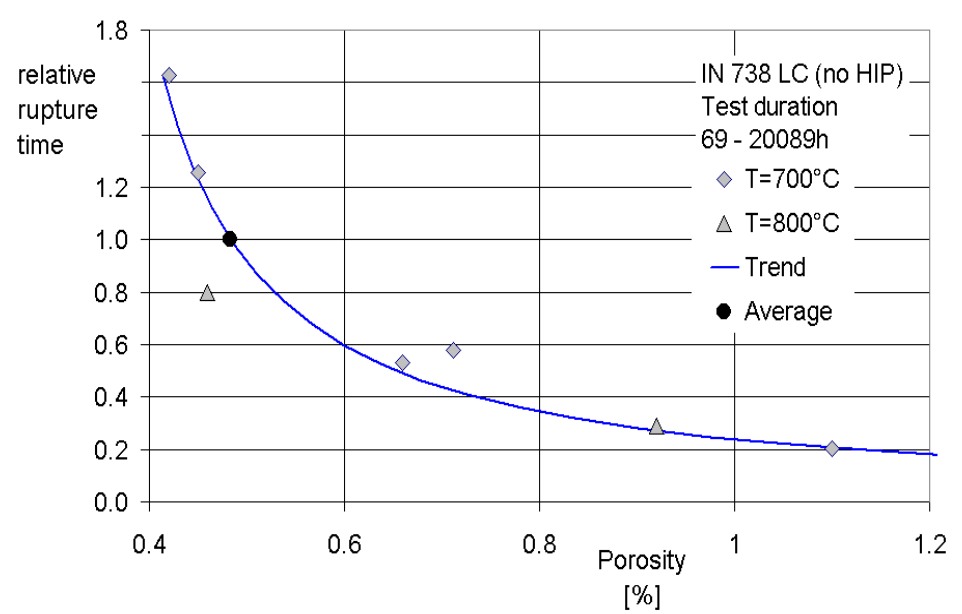

Fig. 14. Creep rupture time vs. porosity in micrograph. The grain size, differing from specimen to specimen, is of superimposed influence.

\section{Summary}

A weak correlation between the chemical composition and the mechanical properties could be detected. As far as it is possible to assess the position of a specific melt in the scatter band, recommendations for favourable melt compositions could be issued. The found relations do not allow to extrapolate beyond the specified range for the alloying elements and, because property scatter of the individual specimens or work pieces is superimposed, the improvements are only statistically detectable.

The porosity of conventionally cast components can only be influenced to a very limited extend by the casting parameters like the temperatures of the melt and the mould but it is possible to minimise the porosity and increase the homogeneity with a subsequent HIP process. The narrowing of the scatter band, the lifting of the lower scatter boundary and consequently the gain in lifetime are very remarkable. An economic balancing of the additional expenses for a HIP treatment of all structural parts and the substantial improvement of the material, however, looks to be necessary.

\section{References}

[1] CEN/WS 11, Secretariat BSI: Measurement uncertainties in mechanical tests on metallic materials - Part3: The evaluation of uncertainties in creep testing, 2004

[2] Monkman, F. C. , Grant, N. J.: An empirical Relationship between Rupture Life and Minimum Creep Rate in Creep Rupture Tests. Proceedings ASTM 593-620, 1956

[3] Bhadeshia, H. K. D. H.: Neural Networks in Materials Science. ISIJ International, Vol. 39, No. 10, 966-979, 1999

[4] Balos, D., Klenk, A., Maile, K., Müller, W.: Anwendung von neuronalen Netzen zur Ermittlung schmelzenabhängigen Zeitstandverhaltens. 29. Vortragsveranstaltung FVW/FVHT, Tagungsband S.137-148, Stahlinstitut VDEh, Düsseldorf, 2006

[5] Stein, K.: Die Gefügeausbildung und ihr Einfluss auf die mechanischen Eigenschaften der Nickelbasislegierung IN738LC in Abhängigkeit von gießtechnischer Erzeugung und Nachbehandlung. Diss. TH Darmstadt, 1983

[6] Müller, F., Scholz, A., Berger, C.: Creep Crack Behaviour of a Course Grain Nickel-base Super-Alloy. Submitted to: High Temperature Defect Assessment (HIDA) - 5 conference, University of Surrey, Guildford, 23-25. June 2010 